\title{
A Study of Relation Between Robustness of Parameter Estimation and Model Order in System Identification - Revised
}

\author{
Mitsuru Matsubara \\ E-mail: mbv707fs@hotmail.com
}

\begin{abstract}
In this paper, for the purpose of evaluating the degradation level of the performance in application with the estimated parametric model in which the estimated parameters have the disturbance, we consider the relation between the robustness of parameter estimation and the model order. In this paper, the robustness of the estimated parameter is defined as the degradation level of the performance. After the application performance cost function is derived, the gradient at the estimated parameter is focused to evaluate the disturbance level of the performance in the application. As a result, when identifying the model with the true structure by maximum-likelihood method, the disturbance level is simply given as the function of the model order. And it is shown that the larger the model order is, the more the estimated parameter lacks the robustness in the application. In addition, the relation between the robustness and a information criterion is introduced.
\end{abstract}

\section{Introduction}

In application using parametric model, to design the application which makes its performance the best, a number of candidate parametric models are generally prepared by estimating them from two or more data sets. When the applications are respectively designed by using those estimated parametric models, the disturbance of the performance of those applications arises due to the disturbance of those data sets(Fig. 1). In the case where the disturbance of the performance is small, then any estimated parametric model can be employed to design the application. However, in the case where the disturbance is large, then the application performance designed by one of the estimated parametric models may not desirable for various data sets. In this paper, the robustness of the estimated parameter of the parametric model shall be defined as the degradation level of the performance caused by such disturbance. Namely, if the degradation of the performance is more small for such disturbance, then the estimated parame- ter is explained as robust. Then, the goal of this study is to clarify what is the factor that makes the disturbance large, i.e., is to clarify what is the factor that reduces the robustness of the estimated parameter. In this framework, the purpose of this paper is to clarify that one of considering factors is the model order.

In parameter estimation of the model, generally, it is important to understand what makes an identification problem difficult. The studies which evaluate the cost of the identification to the system complexity which influences the difficulty of the identification have been done in $[1,2]$. Since the model order is of course one of the causes which make the system complex, the robustness which will be connected to the model order in this paper is similar concept to the cost of the identification to the system complexity. Moreover, the robustness defined in this paper has the similar concept to the penalty term of IC(Information Criterion), the relation between the robustness and the penalty terms of AIC(Akaike's information Criterion)[3], and the relation between the robustness and the penalty terms of TIC(Takeuchi's Information Criterion) [4] are formulated in this paper.

As a result, when estimating the parameter of the model with the true structure by maximum-likelihood method, the disturbance level is simply given as the function of the model order. And it is shown that the larger the model order is, the more the estimated parameter lacks the robustness in the application. In addition, in the case where the model does not have the true structure, the disturbance level is also simply given as the function of the model order, under the assumption that the application adopts normal linear regression model.

\section{Problem Settings}

Let the true system be represented by the discretetime causal linear time-invariant system:

$$
y(k)=G_{0}(q) u(k)+w_{0}(k)
$$

where $G_{0}(q)$ is the true plant, $y, u, w_{0}$ are the output, the input and the output disturbance respectively, and 


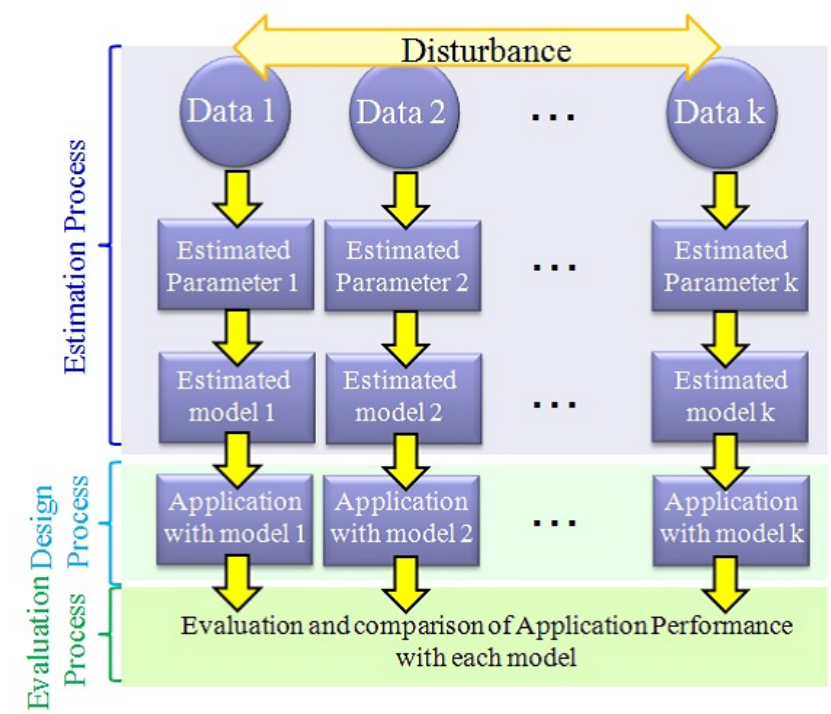

Fig. 1: Disturbance of the application performance in the design process

$q$ denotes the delay operator. In this paper, it is assumed that the objective system can be modeled as follows:

$$
y(k)=G(q, \boldsymbol{\theta}) u(k)+w(k), \quad k=1,2, \cdots, N,
$$

where $w$ is a white noise with mean zero, $\boldsymbol{\theta} \in \mathbb{R}^{p}(\boldsymbol{\theta} \subset$ $\Theta$ ) denotes the model parameter, and $N$ denotes the sample number.

The application performance cost function to the estimated parametric model is defined as follows:

$$
V(\boldsymbol{\theta})=\left[F(\boldsymbol{\theta})-F\left(\boldsymbol{\theta}_{0}\right)\right]^{2},
$$

where $F(\cdot)$ is the arbitrary scalar application cost function. Since $V(\boldsymbol{\theta}) \geq 0$ with minimum $V\left(\boldsymbol{\theta}_{0}\right)=\mathbf{0}, \boldsymbol{\theta}=\boldsymbol{\theta}_{0}$ is the intended condition in the application.

\section{Example:}

Consider the state feedback $\mathcal{H}_{2}$ control problem to the following continuous-time plant model $\mathcal{M}$ :

$$
\mathcal{M}(\boldsymbol{\theta}) \quad: \quad G(s, \boldsymbol{\theta})=C(s I-A)^{-1} B
$$

where $A \in \mathbb{R}^{p \times p}, B \in \mathbb{R}^{p \times 1}, C \in \mathbb{R}^{1 \times p}, s$ denotes Laplace transform variable, $\boldsymbol{\theta}$ is the parameter vector constructed by $(A, B, C)$, and it is assumed that $(A, B)$ and $(A, C)$ are stabilizable and detectable, respectively. Let $X=X^{\mathrm{T}} \geq 0$ be the stabilizing solution to the algebraic Riccati equation:

$$
A^{\mathrm{T}} X+X A-X B B^{\mathrm{T}} X+C^{\mathrm{T}} C=O .
$$

Since the solution becomes the function of $\boldsymbol{\theta}$, let $X$ be $X(\boldsymbol{\theta})$. Then, the optimal state feedback gain $K_{\mathrm{opt}}$ is given as:

$$
K_{\mathrm{opt}}=-B^{\mathrm{T}} X(\boldsymbol{\theta}),
$$

the application performance cost function $F(\boldsymbol{\theta})$ is also defined as the best achievable control performance function $H$ :

$$
F(\boldsymbol{\theta})=H(C(\mathcal{M}(\boldsymbol{\theta})), \mathcal{M}(\boldsymbol{\theta}))=\operatorname{tr}\left\{B X^{\mathrm{T}}(\boldsymbol{\theta}) B\right\},
$$

where $\operatorname{tr}\{\cdot\}$ denotes trace of matrix $\{\cdot\}$ and $C(\mathcal{M}(\boldsymbol{\theta}))$ denotes the controller which is designed based on the plant model $\mathcal{M}(\boldsymbol{\theta})$. In this example, since the controller which is designed to $\mathcal{M}\left(\boldsymbol{\theta}_{0}\right)$ can derive the best performance to the objective system, the parameter $\boldsymbol{\theta}$ which minimizes $V(\boldsymbol{\theta})$ is $\boldsymbol{\theta}_{0}$.

In (3), the larger the norm of the gradient on $V\left(\hat{\boldsymbol{\theta}}_{N}\right)$ at a estimated parameter $\hat{\boldsymbol{\theta}}_{N}$ is, the more the application performance cost with $\hat{\boldsymbol{\theta}}_{N}$ increases, and the more the disturbance of the application performance cost becomes large when the estimated parameter $\hat{\boldsymbol{\theta}}_{N}$ disturbs. This means that even if $\boldsymbol{\theta}_{0} \approx \hat{\boldsymbol{\theta}}_{N}$ is satisfied, when the norm of the gradient on $V\left(\hat{\boldsymbol{\theta}}_{N}\right)$ at a estimated parameter $\hat{\boldsymbol{\theta}}_{N}$ is large, the models with such disturbed estimates $\hat{\boldsymbol{\theta}}_{N}$ lack the robustness in the application. For the reason, in this paper, the robustness of the estimated parameter shall be defined by the norm of the gradient on $V\left(\hat{\boldsymbol{\theta}}_{N}\right)$ at a estimated parameter $\hat{\boldsymbol{\theta}}_{N}$.

\section{Assumptions}

Here, the following assumptions are introduced.

(i) $\boldsymbol{\theta}_{0} \in \mathbb{R}^{p}\left(\boldsymbol{\theta}_{0} \subset \Theta\right)$ is defined such that the following equation can be satisfied:

$$
\int g(x) \frac{\partial \log f(x \mid \boldsymbol{\theta})}{\partial \boldsymbol{\theta}} d x=\mathbf{0}, \quad \boldsymbol{\theta} \in \mathbb{R}^{p}
$$

(ii) Let $g(x)$ be true distribution, and the model $\mathcal{M}(\boldsymbol{\theta})$ with true model structure $(p=n)$ can give $g(x)=f\left(x \mid \boldsymbol{\theta}_{0}\right)$ in $\boldsymbol{\theta}=\boldsymbol{\theta}_{0} \in \mathbb{R}^{n}$.

(iii) The model with the true parameter $\boldsymbol{\theta}_{0} \in \mathbb{R}^{n}$ which has true model structure $(p=n)$ sets the application cost to the best.

(iv) The application $\operatorname{cost} F(\boldsymbol{\theta})$ is monotonically increase by increasing of identification cost.

(v) The identification cost is defined as log-likelihood.

From (v), $\hat{\boldsymbol{\theta}}_{N}$ means maximum likelihood(ML) estimate, and from (iv) and (v), we define $F(\cdot)$ as follows:

$$
\text { case1: } F(\boldsymbol{\theta}) \equiv \log f\left(X_{N} \mid \boldsymbol{\theta}\right),
$$


case2 : $F(\boldsymbol{\theta}) \equiv N \mathrm{E}_{G(z)}\{\log f(Z \mid \boldsymbol{\theta})\} \equiv \bar{F}(\boldsymbol{\theta})$,

where $X_{N}=\{x\}$ denotes the data set which be observed by present time from the past, $Z=\{z\}$ denotes unknown future data set, and $\mathrm{E}_{G(z)}\{\cdot\}$ means the expectation of $\{\cdot\}$ based on the distribution of $g(z)$. By these assumptions, the degradation level of the application performance can be evaluated by the degradation level of the identification cost for each case.

\section{The relation between the robustness of the estimated parameter and the model order}

\subsection{Case 1}

In this subsection, the application performance cost function $V(\boldsymbol{\theta})$ for the observed data $X_{N}$ is derived.

Consider a second order Taylor series approximation to $\log f\left(X_{N} \mid \boldsymbol{\theta}\right)$ around $\hat{\boldsymbol{\theta}}_{N} \in \mathbb{R}^{p}$ :

$$
\begin{aligned}
\log f\left(X_{N} \mid \boldsymbol{\theta}\right) & =\log f\left(X_{N} \mid \hat{\boldsymbol{\theta}}_{N}\right) \\
& +\frac{1}{2}\left(\boldsymbol{\theta}-\hat{\boldsymbol{\theta}}_{N}\right)^{\mathrm{T}} \frac{\partial^{2} \log f\left(X_{N} \mid \hat{\boldsymbol{\theta}}_{N}\right)}{\partial \boldsymbol{\theta} \partial \boldsymbol{\theta}^{\mathrm{T}}}\left(\boldsymbol{\theta}-\hat{\boldsymbol{\theta}}_{N}\right),(9)
\end{aligned}
$$

where $\partial \log f\left(X_{N} \mid \hat{\boldsymbol{\theta}}_{N}\right) / \partial \boldsymbol{\theta}=0$. Here, the measured data set $W=\left\{w(k)\left(=y(k)-G_{0}(q) u(k)\right)\right\}$ is generated in accordance with true distribution $g(z)$, and define $X_{N}$ as $W$. Then, by the law of large numbers and $\hat{\boldsymbol{\theta}}_{N} \rightarrow$ $\boldsymbol{\theta}_{0}(N \rightarrow \infty)$ which are the property of ML estimate $\hat{\boldsymbol{\theta}}_{N}$, the following result can be obtained:

$$
\begin{gathered}
\frac{1}{N} \frac{\partial^{2} \log f\left(X_{N} \mid \hat{\boldsymbol{\theta}}_{N}\right)}{\partial \boldsymbol{\theta} \partial \boldsymbol{\theta}^{\mathrm{T}}}=\frac{1}{N} \sum_{l=1}^{N} \frac{\partial^{2} \log f\left(w(l) \mid \hat{\boldsymbol{\theta}}_{N}\right)}{\partial \boldsymbol{\theta} \partial \boldsymbol{\theta}^{\mathrm{T}}} \\
\rightarrow J\left(\boldsymbol{\theta}_{0}\right), \quad N \rightarrow \infty \\
J\left(\boldsymbol{\theta}_{0}\right)=-\left.\int g(x) \frac{\partial^{2} \log f\left(x \mid \boldsymbol{\theta}_{0}\right)}{\partial \boldsymbol{\theta} \partial \boldsymbol{\theta}^{\mathrm{T}}}\right|_{\boldsymbol{\theta}=\boldsymbol{\theta}_{0}} d x
\end{gathered}
$$

From (10) and (11), (9) becomes approximately as follows:

$$
\begin{aligned}
\log f\left(X_{N} \mid \boldsymbol{\theta}_{0}\right) & -\log f\left(X_{N} \mid \hat{\boldsymbol{\theta}}_{N}\right) \\
& =-\frac{N}{2}\left(\boldsymbol{\theta}_{0}-\hat{\boldsymbol{\theta}}_{N}\right)^{\mathrm{T}} J\left(\boldsymbol{\theta}_{0}\right)\left(\boldsymbol{\theta}_{0}-\hat{\boldsymbol{\theta}}_{N}\right)
\end{aligned}
$$

Then, from (3) and (12), $V$ with the ML estimate $\hat{\boldsymbol{\theta}}_{N}$ is approximately:

$$
\begin{aligned}
V\left(\hat{\boldsymbol{\theta}}_{N}\right) & =\left[\log f\left(X_{N} \mid \hat{\boldsymbol{\theta}}_{N}\right)-\log f\left(X_{N} \mid \boldsymbol{\theta}_{0}\right)\right]^{2} \\
& =\frac{N^{2}}{4}\left[\left(\boldsymbol{\theta}_{0}-\hat{\boldsymbol{\theta}}_{N}\right)^{\mathrm{T}} J\left(\boldsymbol{\theta}_{0}\right)\left(\boldsymbol{\theta}_{0}-\hat{\boldsymbol{\theta}}_{N}\right)\right]^{2} .
\end{aligned}
$$

Our purpose is to evaluate the norm of the gradient of $V(\boldsymbol{\theta})$ at $\boldsymbol{\theta}=\hat{\boldsymbol{\theta}}_{N}$ to evaluate the robustness of the estimated parameter.
Let $L(\boldsymbol{\theta})$ be tangent of $V(\boldsymbol{\theta})$ at $\hat{\boldsymbol{\theta}}_{N}$, then $L(\boldsymbol{\theta})$ is given by:

$$
\begin{aligned}
L(\boldsymbol{\theta}) & =\left.\frac{d V(\boldsymbol{\theta})}{d \boldsymbol{\theta}^{\mathrm{T}}}\right|_{\boldsymbol{\theta}=\hat{\boldsymbol{\theta}}_{N}}\left(\boldsymbol{\theta}-\hat{\boldsymbol{\theta}}_{N}\right)+V\left(\hat{\boldsymbol{\theta}}_{N}\right), \\
\frac{d V(\boldsymbol{\theta})}{d \boldsymbol{\theta}^{\mathrm{T}}} & =N^{2}\left(\boldsymbol{\theta}-\boldsymbol{\theta}_{0}\right)^{\mathrm{T}} J\left(\boldsymbol{\theta}_{0}\right)\left(\boldsymbol{\theta}-\boldsymbol{\theta}_{0}\right)^{\mathrm{T}} J\left(\boldsymbol{\theta}_{0}\right)\left(\boldsymbol{\theta}-\boldsymbol{\theta}_{0}\right)
\end{aligned}
$$

Consider the intercept of $L(\boldsymbol{\theta})$ at $\boldsymbol{\theta}_{0}$, then from $(13) \sim$ (15), $L\left(\boldsymbol{\theta}_{0}\right)$ is given as follows:

$$
\begin{aligned}
L\left(\boldsymbol{\theta}_{0}\right) & =\left.\frac{d V(\boldsymbol{\theta})}{d \boldsymbol{\theta}^{\mathrm{T}}}\right|_{\boldsymbol{\theta}=\hat{\boldsymbol{\theta}}_{N}}\left(\boldsymbol{\theta}_{0}-\hat{\boldsymbol{\theta}}_{N}\right)+V\left(\hat{\boldsymbol{\theta}}_{N}\right) \\
& =-N^{2}\left[\left(\hat{\boldsymbol{\theta}}_{N}-\boldsymbol{\theta}_{0}\right)^{\mathrm{T}} J\left(\boldsymbol{\theta}_{0}\right)\left(\hat{\boldsymbol{\theta}}_{N}-\boldsymbol{\theta}_{0}\right)\right]^{2}+V\left(\hat{\boldsymbol{\theta}}_{N}\right) \\
& =-4 V\left(\hat{\boldsymbol{\theta}}_{N}\right)+V\left(\hat{\boldsymbol{\theta}}_{N}\right)=-3 V\left(\hat{\boldsymbol{\theta}}_{N}\right) .
\end{aligned}
$$

This means that when a point $\hat{\boldsymbol{\theta}}_{N}$ is fixed, the norm of the gradient of $V(\boldsymbol{\theta})$ at $\hat{\boldsymbol{\theta}}_{N}$ can evaluate by using $V\left(\hat{\boldsymbol{\theta}}_{N}\right)$. Therefore, the robustness of the estimated parameter $\hat{\boldsymbol{\theta}}_{N}$ in the application shall be evaluated by $V\left(\hat{\boldsymbol{\theta}}_{N}\right)$.

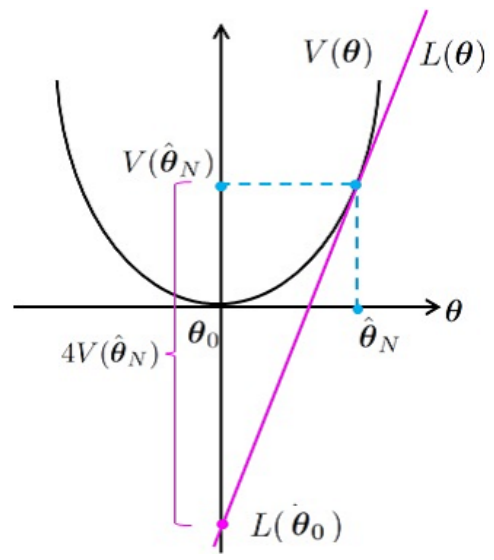

Fig. 2: Tangent $L(\boldsymbol{\theta})$ and the intercept $L\left(\boldsymbol{\theta}_{0}\right)$

\subsection{Case 2}

In this subsection, the application performance cost function $V(\boldsymbol{\theta})$ for the unknown future data $Z$ is derived.

Consider a second order Taylor series approximation to $\mathrm{E}_{G(z)}\{\log f(Z \mid \boldsymbol{\theta})\}$ around $\boldsymbol{\theta}_{0} \in \mathbb{R}^{p}$ :

$$
\begin{aligned}
\mathrm{E}_{G(z)}\left\{\log f\left(Z \mid \hat{\boldsymbol{\theta}}_{N}\right)\right\} & =\mathrm{E}_{G(z)}\left\{\log f\left(Z \mid \boldsymbol{\theta}_{0}\right)\right\} \\
& -\frac{1}{2}\left(\hat{\boldsymbol{\theta}}_{N}-\boldsymbol{\theta}_{0}\right)^{\mathrm{T}} J\left(\boldsymbol{\theta}_{0}\right)\left(\hat{\boldsymbol{\theta}}_{N}-\boldsymbol{\theta}_{0}\right) .
\end{aligned}
$$

Then, from (3), in this case, $V$ with the ML estimate $\hat{\boldsymbol{\theta}}_{N}$ is approximately:

$$
\begin{aligned}
V\left(\hat{\boldsymbol{\theta}}_{N}\right) & =\left[N \mathrm{E}_{G(z)}\left\{\log f\left(Z \mid \hat{\boldsymbol{\theta}}_{N}\right)\right\}-N \mathrm{E}_{G(z)}\left\{\log f\left(Z \mid \boldsymbol{\theta}_{0}\right)\right\}\right]^{2} \\
& =\frac{N^{2}}{4}\left[\left(\hat{\boldsymbol{\theta}}_{N}-\boldsymbol{\theta}_{0}\right)^{\mathrm{T}} J\left(\boldsymbol{\theta}_{0}\right)\left(\hat{\boldsymbol{\theta}}_{N}-\boldsymbol{\theta}_{0}\right)\right]^{2} \equiv \bar{V}\left(\hat{\boldsymbol{\theta}}_{N}\right) .(18)
\end{aligned}
$$

Since (18) is equivalent to (13), also in this case, the robustness of the estimated parameter in the application shall be evaluated by $V\left(\hat{\boldsymbol{\theta}}_{N}\right)$ as well as case 1 . 


\subsection{The relation between the robustness of the} estimated parameter and the model order

To evaluate the gradient by using $V\left(\hat{\boldsymbol{\theta}}_{N}\right)$, for each case, the estimated parameter $\hat{\boldsymbol{\theta}}_{N} \in \mathbb{R}^{p}$ has to be fixed because the estimate $\hat{\boldsymbol{\theta}}_{N}$ is the function of the data set which can be observed by the present time from the past, i.e., $\hat{\boldsymbol{\theta}}_{N}(\boldsymbol{w})$, and also the estimated $\hat{\boldsymbol{\theta}}_{N}(\boldsymbol{w})$ actually has the disturbance. Thus, $V\left(\hat{\boldsymbol{\theta}}_{N}\right)$ for each case shall be re-defined as follows:

$$
V_{\text {ave }}\left(\hat{\boldsymbol{\theta}}_{N}\right) \equiv \mathrm{E}_{G(w)}\left\{V\left(\hat{\boldsymbol{\theta}}_{N}(\boldsymbol{w})\right)\right\},
$$

where $\mathrm{E}_{G(w)}\{\cdot\}$ denotes the average to the measured data set $W=\{w\}$.

It is known that ML estimate has the following property:

$$
\begin{aligned}
Q \equiv N\left(\hat{\boldsymbol{\theta}}_{N}-\boldsymbol{\theta}_{0}\right)^{\mathrm{T}} J\left(\boldsymbol{\theta}_{0}\right) I^{-1}\left(\boldsymbol{\theta}_{0}\right) J\left(\boldsymbol{\theta}_{0}\right)\left(\hat{\boldsymbol{\theta}}_{N}-\boldsymbol{\theta}_{0}\right) \\
\sim \chi^{2}(p), \quad N \rightarrow \infty,
\end{aligned}
$$

where $I\left(\boldsymbol{\theta}_{0}\right)$ denotes Fisher's information matrix:

$$
I\left(\boldsymbol{\theta}_{0}\right)=\left.\int g(w) \frac{\partial \log f(w \mid \boldsymbol{\theta})}{\partial \boldsymbol{\theta}} \frac{\partial \log f(w \mid \boldsymbol{\theta})}{\partial \boldsymbol{\theta}^{\mathrm{T}}}\right|_{\boldsymbol{\theta}=\boldsymbol{\theta}_{0}} d w .
$$

If $\boldsymbol{\theta}_{0}$ which satisfies $g(w)=f\left(w \mid \boldsymbol{\theta}_{0}\right)$ exists in $\Theta$, then the relation $I\left(\boldsymbol{\theta}_{0}\right)=J\left(\boldsymbol{\theta}_{0}\right),(p=n)$ is obtained. As a result, under this condition, $V_{\text {ave }}\left(\hat{\boldsymbol{\theta}}_{N}\right)$ becomes simple result as shown below(eq:(22) and (23)).

The expectation and the variance of the random variable which is distributed according to the chi-squared distribution with $p$ degrees of freedom is $p$ and $2 p$ respectively. Therefore, based on (20), for case 1 , the following equation is derived:

$$
\begin{aligned}
V_{\text {ave }}\left(\hat{\boldsymbol{\theta}}_{N}\right) & =\mathrm{E}_{G(w)}\left\{V\left(\hat{\boldsymbol{\theta}}_{N}(\boldsymbol{w})\right)\right\} \\
& =\mathrm{E}_{G(w)}\left\{\frac{N^{2}}{4}\left[\left(\hat{\boldsymbol{\theta}}_{N}-\boldsymbol{\theta}_{0}\right)^{\mathrm{T}} J\left(\boldsymbol{\theta}_{0}\right)\left(\hat{\boldsymbol{\theta}}_{N}-\boldsymbol{\theta}_{0}\right)\right]^{2}\right\} \\
& =\frac{1}{4}\left[\operatorname{Var}_{G(w)}\{Q\}+\left(\mathrm{E}_{G(w)}\{Q\}\right)^{2}\right] \\
& =\frac{1}{4} p(p+2), \quad(p=n, p \geq 1) .
\end{aligned}
$$

For the case 2, also the same result is obtained as follows:

$$
\begin{aligned}
V_{\mathrm{ave}}\left(\hat{\boldsymbol{\theta}}_{N}\right) & =\mathrm{E}_{G(w)}\left\{\bar{V}\left(\hat{\boldsymbol{\theta}}_{N}(\boldsymbol{w})\right)\right\} \\
& =\frac{1}{4} p(p+2), \quad(p=n, p \geq 1) .
\end{aligned}
$$

From (22) and (23), in the case where model $\mathcal{M}(\boldsymbol{\theta})$ can have true structure, i.e., model order is $p=n$, we obtain the fact that the larger model order is, the more the application performance cost function $V_{\text {ave }}\left(\hat{\boldsymbol{\theta}}_{N}\right)$ monotonically increase for each case. In other words, in the case where identifying $\mathcal{M}(\boldsymbol{\theta})$ with true structure by ML method, the larger model scale is, the more the disturbance level of the application performance with the model becomes large, even if $\boldsymbol{\theta}_{0} \approx \hat{\boldsymbol{\theta}}_{N}$ is satisfied. As a result, we obtain the fact that the estimated parameter lacks the robustness in above case $(p=n)$.

On the other hand, if $\boldsymbol{\theta}_{0}$ which satisfied $g(w)=$ $f\left(w \mid \boldsymbol{\theta}_{0}\right)$ does not exist for all $\boldsymbol{\theta} \subset \Theta$ due to $p \neq n$, the relation $I\left(\boldsymbol{\theta}_{0}\right) \neq J\left(\boldsymbol{\theta}_{0}\right)$ is held. Then, $V_{\text {ave }}\left(\hat{\boldsymbol{\theta}}_{N}\right)$ becomes complex result as shown below.

It is known that $\sqrt{N} \Delta \boldsymbol{\theta} \equiv \sqrt{N}\left(\hat{\boldsymbol{\theta}}_{N}-\boldsymbol{\theta}_{0}\right) \in \mathbb{R}^{p}$ has asymptotic distribution[3]:

$$
\begin{aligned}
\sqrt{N} \Delta \boldsymbol{\theta} & \equiv \sqrt{N}\left(\hat{\boldsymbol{\theta}}_{N}-\boldsymbol{\theta}_{0}\right) \\
& \sim \mathcal{N}_{p}\left(\mathbf{0}, J^{-1}\left(\boldsymbol{\theta}_{0}\right) I\left(\boldsymbol{\theta}_{0}\right) J^{-1}\left(\boldsymbol{\theta}_{0}\right)\right), \quad N \rightarrow \infty .
\end{aligned}
$$

$V_{\text {ave }}\left(\hat{\boldsymbol{\theta}}_{N}\right)$ can be also represented as follows:

$$
\begin{aligned}
V_{\text {ave }}\left(\hat{\boldsymbol{\theta}}_{N}\right) & =\frac{1}{4} \sum_{i=1}^{p} \sum_{j=1}^{p} \sum_{k=1}^{p} \sum_{l=1}^{p} J_{i j}\left(\boldsymbol{\theta}_{0}\right) J_{k l}\left(\boldsymbol{\theta}_{0}\right) \\
& \times \mathrm{E}_{G(w)}\left\{\sqrt{N} \Delta \theta_{i} \sqrt{N} \Delta \theta_{j} \sqrt{N} \Delta \theta_{k} \sqrt{N} \Delta \theta_{l}\right\} .
\end{aligned}
$$

Besed on (24), since $\sqrt{N} \Delta \theta_{i}$ for $\forall i$ are asymptotically jointly normal with mean zero, 4 th order moment[5] in (25) is given by:

$$
\begin{aligned}
& \mathrm{E}_{G(w)}\left\{\sqrt{N} \Delta \theta_{i} \sqrt{N} \Delta \theta_{j} \sqrt{N} \Delta \theta_{k} \sqrt{N} \Delta \theta_{l}\right\}= \\
& \quad \mathrm{E}_{G(w)}\left\{\sqrt{N} \Delta \theta_{i} \sqrt{N} \Delta \theta_{j}\right\} \mathrm{E}_{G(w)}\left\{\sqrt{N} \Delta \theta_{k} \sqrt{N} \Delta \theta_{l}\right\} \\
& +\mathrm{E}_{G(w)}\left\{\sqrt{N} \Delta \theta_{i} \sqrt{N} \Delta \theta_{k}\right\} \mathrm{E}_{G(w)}\left\{\sqrt{N} \Delta \theta_{j} \sqrt{N} \Delta \theta_{l}\right\} \\
& +\mathrm{E}_{G(w)}\left\{\sqrt{N} \Delta \theta_{i} \sqrt{N} \Delta \theta_{l}\right\} \mathrm{E}_{G(w)}\left\{\sqrt{N} \Delta \theta_{j} \sqrt{N} \Delta \theta_{k}\right\},
\end{aligned}
$$$$
N \rightarrow \infty
$$

Consequently, from (26), $V_{\text {ave }}\left(\hat{\boldsymbol{\theta}}_{N}\right)$ becomes as follows:

$$
V_{\text {ave }}\left(\hat{\boldsymbol{\theta}}_{N}\right)=\frac{3}{4}\left(\operatorname{tr}\left\{I\left(\boldsymbol{\theta}_{0}\right) J^{-1}\left(\boldsymbol{\theta}_{0}\right)\right\}\right)^{2}-h(I, J),
$$

where $\operatorname{tr}\{\cdot\}$ denotes trace of matrix $\{\cdot\}$, and $h$ is the function of $I\left(\boldsymbol{\theta}_{0}\right)$ and $J\left(\boldsymbol{\theta}_{0}\right)$ :

$$
\begin{aligned}
& h(I, J)= \\
& 2 \sum_{i=1}^{p} \sum_{j=1}^{p} \sum_{\substack{k=1 \\
j<k}}^{p} \sum_{\substack{l=1 \\
i<l}}^{p} \operatorname{det}\left[\Gamma\left(\begin{array}{cc}
j & k \\
i & l
\end{array}\right) \cdot J\left(\boldsymbol{\theta}_{0}\right)\left(\begin{array}{cc}
j & k \\
i & l
\end{array}\right)\right] \\
& \Gamma \equiv N E_{G(w)}\left\{\left(\hat{\boldsymbol{\theta}}_{N}-\boldsymbol{\theta}_{0}\right)\left(\hat{\boldsymbol{\theta}}_{N}-\boldsymbol{\theta}_{0}\right)^{\mathrm{T}}\right\} \\
& \rightarrow J^{-1}\left(\boldsymbol{\theta}_{0}\right) I\left(\boldsymbol{\theta}_{0}\right) J^{-1}\left(\boldsymbol{\theta}_{0}\right), \quad N \rightarrow \infty,
\end{aligned}
$$

and $\mathcal{A}(\cdot)$ denotes a minor of a matrix $\mathcal{A} \in \mathbb{R}^{r \times s}$ as defined by:

$$
\mathcal{A}\left(\begin{array}{cc}
j & k \\
i & l
\end{array}\right) \equiv\left[\begin{array}{cc}
a_{i j} & a_{i k} \\
a_{l j} & a_{l k}
\end{array}\right] \quad \in \mathbb{R}^{2 \times 2}
$$

Unfortunately, it is not easy to clarify analytically the relation between the robustness and the model order based on (27). And so, in Section 6, under the 
assumption that the application adopts normal linear regression model, the relation between the robustness and the model order in the case $p \neq n$ is clarified via the definition of IC's penalty term introduced in next section.

\section{The relation between the robustness of the estimated parameter and the penalty term of information criterion}

This section provides the relation between the robustness of the estimated parameter and the penalty term of information criterion.

The penalty term of IC is generally defined as follows:

$$
\begin{array}{r}
B_{\mathrm{IC}}=2 \mathrm{E}_{G(w)}\left\{\log f\left(W \mid \hat{\boldsymbol{\theta}}_{N}(\boldsymbol{w})\right)-N \mathrm{E}_{G(z)}\left\{\log f\left(Z \mid \hat{\boldsymbol{\theta}}_{N}(\boldsymbol{w})\right)\right\}\right\}, \\
\hat{\boldsymbol{\theta}}_{N}(\boldsymbol{w}) \in \mathbb{R}^{p} .(31)
\end{array}
$$

By the definition of $F$ and $\bar{F}$, the penalty term of IC can be represented as follows:

$$
\begin{aligned}
B_{\mathrm{IC}}= & 2 \mathrm{E}_{G(w)}\left\{F\left(\hat{\boldsymbol{\theta}}_{N}(\boldsymbol{w})\right)-F\left(\boldsymbol{\theta}_{0}\right)\right\} \\
& +2 \mathrm{E}_{G(w)}\left\{F\left(\boldsymbol{\theta}_{0}\right)-\bar{F}\left(\boldsymbol{\theta}_{0}\right)\right\} \\
& +2 \mathrm{E}_{G(w)}\left\{\bar{F}\left(\boldsymbol{\theta}_{0}\right)-\bar{F}\left(\hat{\boldsymbol{\theta}}_{N}(\boldsymbol{w})\right)\right\}
\end{aligned}
$$

Then, from (32), the penalty term of TIC can be written as follows [3]:

$$
B_{\mathrm{TIC}}=2 \operatorname{tr}\left\{\hat{I} \hat{J}^{-1}\right\}
$$

Since $F\left(\hat{\boldsymbol{\theta}}_{N}\right) \geq F\left(\boldsymbol{\theta}_{0}\right)$ and $\bar{F}\left(\boldsymbol{\theta}_{0}\right) \geq \bar{F}\left(\hat{\boldsymbol{\theta}}_{N}\right)$ from $(12)$ and (17),

$$
\begin{aligned}
\sqrt{V\left(\hat{\boldsymbol{\theta}}_{N}\right)} & =F\left(\hat{\boldsymbol{\theta}}_{N}(\boldsymbol{w})\right)-F\left(\boldsymbol{\theta}_{0}\right) \\
\sqrt{\bar{V}\left(\hat{\boldsymbol{\theta}}_{N}\right)} & =\bar{F}\left(\boldsymbol{\theta}_{0}\right)-\bar{F}\left(\hat{\boldsymbol{\theta}}_{N}(\boldsymbol{w})\right)
\end{aligned}
$$

are obtained. In addition, since $\mathrm{E}_{G(w)}\left\{F\left(\boldsymbol{\theta}_{0}\right)-\bar{F}\left(\boldsymbol{\theta}_{0}\right)\right\}=$ 0 is satisfied, by using the application performance cost function $V(\boldsymbol{\theta})$, the penalty term of TIC is also given as:

$B_{\mathrm{TIC}}=2 \mathrm{E}_{G(w)}\left\{\sqrt{V\left(\hat{\boldsymbol{\theta}}_{N}\right)}\right\}+2 \mathrm{E}_{G(w)}\left\{\sqrt{\bar{V}\left(\hat{\boldsymbol{\theta}}_{N}\right)}\right\}$.

This result means for all $\hat{\boldsymbol{\theta}}_{N} \subset \Theta \in \mathbb{R}^{p}$ that the larger the $V\left(\hat{\boldsymbol{\theta}}_{N}\right)$ which evaluates the robustness of the estimated parameter is, i.e., the disturbance level of the application performance is large, the more log-likelihood has to be corrected by the penalty term. As a result, this result shows that the larger the penalty term of TIC is, the estimated parameter lacks the robustness in the application.
If $\boldsymbol{\theta}_{0}$ which satisfies $g(w)=f\left(w \mid \boldsymbol{\theta}_{0}\right)$ exists in $\Theta$, then the relation $I\left(\boldsymbol{\theta}_{0}\right)=J\left(\boldsymbol{\theta}_{0}\right),(p=n)$ is obtained. Therefore, from (12), (17) and (20), the penalty term simply becomes as follows:

$$
\begin{aligned}
B_{\mathrm{AIC}} & =2 \mathrm{E}_{G(w)}\left\{\sqrt{V\left(\hat{\boldsymbol{\theta}}_{N}\right)}\right\}+2 \mathrm{E}_{G(w)}\left\{\sqrt{\bar{V}\left(\hat{\boldsymbol{\theta}}_{N}\right)}\right\} \\
& =\mathrm{E}_{G(w)}\{Q\}+\mathrm{E}_{G(w)}\{Q\} \\
& =2 n .
\end{aligned}
$$

(37) is the penalty term of AIC exactly. In the case where model $\mathcal{M}(\boldsymbol{\theta})$ can have true structure, i.e., the model order is $p=n$, this result means that the larger the model order is, i.e., the structure of true model is complex, the more the disturbance level of the application performance becomes large, and the more loglikelihood has the bias to log-likelihood of the true model by the penalty term. As a result, this result shows that the larger the model order is, the estimated parameter lacks the robustness in the application.

\section{Normal Linear Regression Case}

In this section, we consider the case where the application adopts normal linear regression model, namely the case where the model is given as follows:

$$
\boldsymbol{y}=U \boldsymbol{\theta}+\boldsymbol{e}, \quad \boldsymbol{e} \sim \mathcal{N}\left(\mathbf{0}, \sigma^{2} I_{N}\right),
$$

where $\boldsymbol{y} \in \mathbb{R}^{N}$ and $U \in \mathbb{R}^{N \times n}$ denote output vector and input matrix respectively, and the element $e_{i}$ of $\boldsymbol{e}$ is independent identically distributed normal random variables with mean zero and variance $\sigma^{2}$. Here, we assume that true model is explained by (38), and its parameter is $\left(\boldsymbol{\theta}_{0}, \sigma_{0}^{2}\right)$.

The ML estimate of $\left(\boldsymbol{\theta}, \sigma^{2}\right)$ in $(38)$ is:

$\hat{\boldsymbol{\theta}}=\left(U^{\mathrm{T}} U\right)^{-1} U^{\mathrm{T}} \boldsymbol{y}, \quad \hat{\sigma}^{2}=\frac{1}{N}(\boldsymbol{y}-U \hat{\boldsymbol{\theta}})^{\mathrm{T}}(\boldsymbol{y}-U \hat{\boldsymbol{\theta}})$.

By using aboves, the maximum log-likelihood is given as follows:

$$
\log f(\boldsymbol{y} \mid \hat{\boldsymbol{\theta}})=-\frac{N}{2} \log f\left(2 \pi \hat{\sigma}^{2}\right)-\frac{N}{2}
$$

On the other hand, in $\boldsymbol{z}$ which is mutually independent to $\boldsymbol{y}$, the following equation is derived:

$$
\begin{aligned}
& N \mathrm{E}_{G(\boldsymbol{z})}\{\log f(\boldsymbol{z} \mid \hat{\boldsymbol{\theta}}(\boldsymbol{y}))\}= \\
& -\frac{N}{2}\left\{\log f\left(2 \pi \hat{\sigma}^{2}\right)+\frac{\sigma_{o}^{2}}{\hat{\sigma}^{2}}+\frac{\left(\boldsymbol{\theta}_{0}-\hat{\boldsymbol{\theta}}\right)^{\mathrm{T}} U^{\mathrm{T}} U\left(\boldsymbol{\theta}_{0}-\hat{\boldsymbol{\theta}}\right)}{N \hat{\sigma}^{2}}\right\} .
\end{aligned}
$$

By substituting (40) and (41) for (31), the penalty term of IC(or TIC) is obtained as follows [6]

$$
\begin{aligned}
B_{\mathrm{TIC}} & =2 \mathrm{E}_{G(\boldsymbol{y})}\left\{\log f(\boldsymbol{y} \mid \hat{\boldsymbol{\theta}})-N \mathrm{E}_{G(\boldsymbol{z})}\{\log f(\boldsymbol{z} \mid \hat{\boldsymbol{\theta}})\}\right\}, \\
& =\frac{N(n+1)}{N-n-2}
\end{aligned}
$$


From (36) and (42), in the case where candidate model $\mathcal{M}(\boldsymbol{\theta})$ does not have true structure, i.e., model order is $p \neq n$, we obtain the fact that under the condition $N>>n$, the larger model order is, the more the application performance cost function $V_{\text {ave }}(\hat{\boldsymbol{\theta}})$ and $\bar{V}_{\text {ave }}(\hat{\boldsymbol{\theta}})$ monotonically increase. In other words, in the case where identifying $\mathcal{M}(\boldsymbol{\theta})$ including true structure by ML method, the larger model scale is, the more the disturbance level of the application performance with the model becomes large, even if $\boldsymbol{\theta}_{0} \approx \hat{\boldsymbol{\theta}}_{N}$ is satisfied. As a result, we obtain the fact that the estimated parameter lacks the robustness in the case $p \neq n$ under the assumption that the application adopts normal linear regression model.

\section{Similar Representations}

As the relation between the robustness of the estimated parameter in the application and the model order, two similar formulas are already proposed at least. The one is given as follows[2]:

$$
\operatorname{Var}\left\{G\left(e^{j \omega}, \hat{\boldsymbol{\theta}}_{N}\right)\right\} \approx n \frac{\Phi_{w}\left(e^{j \omega}\right)}{N \Phi_{u}\left(e^{j \omega}\right)},
$$

where $\Phi_{w}\left(e^{j \omega}\right)$ and $\Phi_{u}\left(e^{j \omega}\right)$ are noise power spectrum and input power spectrum respectively. This means that the larger the model order $n$ is, the more the disturbance level of the estimated model $G\left(e^{j \omega}, \hat{\boldsymbol{\theta}}_{N}\right)$ increases. Therefore, if the more $G\left(e^{j \omega}, \hat{\boldsymbol{\theta}}_{N}\right)$ is disturbed, the more the disturbance level of the application may be increased.

The other is given as follows:

$$
\begin{array}{r}
\frac{1}{2 \pi} \int_{-\pi}^{\pi} N \Phi_{u}\left(e^{j \omega}\right) \operatorname{Var}\left\{G\left(e^{j \omega}, \hat{\boldsymbol{\theta}}_{N}\right)\right\} d \omega \\
=n \operatorname{Var}\{w(t)\}
\end{array}
$$

There is a water-bed effect for the variance of $G\left(e^{j \omega}, \hat{\boldsymbol{\theta}}_{N}\right)$, i.e., if the variance is made small in some frequency regions it must be large in another region to correct the inequality. In the case where the input power spectrum is invariant, this means that the larger model order $n$ is, the more the disturbance level of the estimated model $G\left(e^{j \omega}, \hat{\boldsymbol{\theta}}_{N}\right)$ is increased. Therefore, if the more $G\left(e^{j \omega}, \hat{\boldsymbol{\theta}}_{N}\right)$ is disturbed, the more the disturbance level of the application may be increased.

If the model order $n$ is fixed in (38), the method minimizing the model variance $\operatorname{Var}\left\{G\left(e^{j \omega}, \hat{\boldsymbol{\theta}}_{N}\right)\right\}$ in the intended frequency regions, which is related to the disturbance level of the application, is to only design the input $\Phi_{u}\left(e^{j \omega}\right)$ appropriately. From such a viewpoint, the method which designs the optimal input in system identification for control design is proposed in $[7,8]$.

\section{Conclusions}

In this paper, for the purpose of evaluating the degradation level of the performance in application with the parametric model in which the estimated parameters have the disturbance, we considered the relation between the robustness of the parameter estimation and the model order. After the application performance cost function was derived, the gradient at the estimated parameter was focused to evaluate the disturbance level of the performance in the application. In this paper, it was shown that when identifying the model with the true structure by ML method, the disturbance level was simply given as the function of the model order. In addition, when identifying the model class including the true structure by ML method, also the disturbance level was given as the function of the model order via the penalty term of IC under the assumption that the application adopts normal linear regression model. As a result, it was shown that the larger the model order is, the more the estimated parameter lacks the robustness in the application for the two case $p=n$ and $p \neq n$. In addition, the relation between the robustness and information criteria was introduced. In future work, we will verify the proposed facts by numerical analysis.

\section{References}

[1] H. Hjalmarsson: From experiment design to closed-loop control, Automatica, Vol.41, no.3, pp.393-438, 2005.

[2] H. Hjalmarsson: System Identification of Complex and Structured Systems, Proc. of the European Conf. 2009, pp.3424-3452, Budapest, Hungary, Aug. 2009.

[3] G. Kitagawa and S. Konishi: Information Criteria(in Japanese), Asakura, Tokyo 2004.

[4] K. Takeuchi: Distribution of Information Statistics and Criteria for Adequacy of Models(in Japanese), Mathematical Sciences, No.153, pp.12-18, 1976.

[5] A. Papoulis and S. U. Pillai: Probability, Random Variables and Stochastic Process, Forth Edition, McGrawHill, 2002.

[6] N. Sugiura: Further Analysis of the data by Akaike's information criterion and finite corrections, Communications in Statistics, Vol.A78, pp.13-26, 1978.

[7] B. Wahlberg, H. Hjalmarsson and M. Annergren: On Optimal Input Design in System Identification for Control, 49th IEEE Conf. on Decision and Control, pp.5548-5553, Atlanta, GA, USA, Dec. 2010.

[8] B. Wahlberg, M. Annergren and C. R. Rojas: On Optimal Input Design for Identification of Output Error Models, 50th IEEE Conf. on Decision and Control, pp.5118-5124, Stockholm, Sweden, Dec. 2011. 